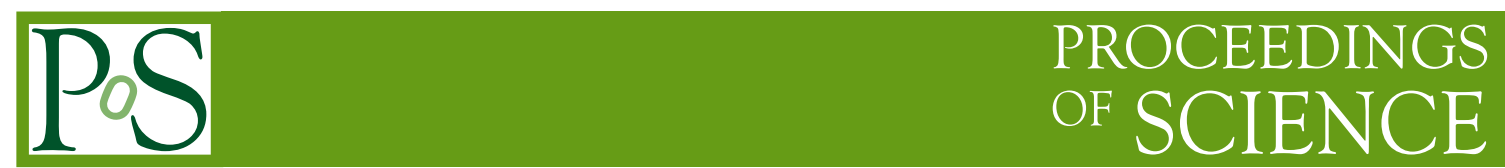

\title{
Weak-coupling expansion of the hot QCD pressure
}

\section{York Schröder*}

Faculty of Physics, University of Bielefeld, D-33501 Bielefeld, Germany

E-mail: yorks@physik.uni-bielefeld.de

We review recent progress made in determining the pressure of hot QCD up to the first nonperturbative term in its weak-coupling expansion.

29th Johns Hopkins Workshop on current problems in particle theory: strong matter in the heavens 1-3 August

Budapest, Hungary

${ }^{*}$ Speaker. 


\section{Introduction}

The pressure of hot $\mathrm{QCD}, p_{\mathrm{QCD}}$, constituting one of the most fundamental thermodynamic observables, has been under theoretical study for several decades now. Being of fundamental importance to cosmology (due to its influence on the cooling rate of the early universe) as well as of potential relevance to heavy ion collisions (through its influence on the thermodynamic expansion rate), $p_{\mathrm{QCD}}$ has been computed using a variety of methods, including lattice Monte-Carlo, weak-coupling and large- $N_{\mathrm{f}}$-methods, to name a few.

Although at large temperatures $T$ asymptotic freedom leads to the expectation that weakcoupling methods are sufficient to accurately describe the deconfined phase, it is well known that the infrared (IR) sector of QCD produces a challenge for perturbation theory, which for the case of the pressure arises at order $g^{6} T^{4}[1]$.

It has however been realized that this challenge can be overcome in an effective theory setup, where the problematic sector is described by a dimensionally reduced theory [2]. The key observation is that thermal (equilibrium) QCD possesses three distinct physical scales, two of them generated dynamically. The contributions to the pressure (and to any other thermodynamic observable) from each of these scales can be obtained from carefully constructing and matching a series of effective theories (for details, see e.g. [3, 4]).

The theories under consideration are (hard) QCD, electrostatic QCD (EQCD) and magnetostatic QCD (MQCD), governing physics on length scales $1 / T$ (the typical scale for a particle in the heat-bath), $1 / g T$ (the dynamically generated screening length for longitudinal gluonic excitations) and $1 / g^{2} T$ (the dynamically generated screening length for transverse gluonic excitations), respectively. While the first two are amenable to perturbative calculations, MQCD is purely nonperturbative and has to be treated on the lattice. Viewing the gauge coupling $g(T)$ as parametrically small (which is certainly justified at asymptotically high temperatures), these three scales are well separated, and can hence be dealt with individually via the effective theory setup. Schematically, for the pressure one can write $p_{\mathrm{QCD}}=p_{E}+p_{M}+p_{G}$, where each contribution depends on the matching scales. This scale-dependence will cancel in the sum, rendering $p_{\mathrm{QCD}}$ a well-defined physical observable.

In this letter, we will give a somewhat condensed account of what we currently know about the different contributions to $p_{\mathrm{QCD}}$.

\section{Status of the QCD pressure}

Below, we specify the contributions to the $\overline{\mathrm{MS}}$ pressure $p_{\mathrm{QCD}}=p_{G}+p_{M}+p_{E}$ [3] from each physical scale individually, for the case of gauge group $\mathrm{SU}\left(N_{\mathrm{c}}\right)$ and $N_{\mathrm{f}}$ quark flavors. We will work at zero quark masses $m_{q_{i}}=0$ and vanishing chemical potentials $\mu_{f}=0$, and display all dependence on the $\overline{\mathrm{MS}}$ scale $\bar{\mu}^{2}=4 \pi e^{-\gamma_{0}} \mu^{2}$ by $L \equiv \ln \frac{\bar{\mu}}{4 \pi T}$. Effects due to finite quark masses $[5,6]$ and chemical potentials [7] are available in the literature, but will not be discussed here.

\subsection{Contributions from the ultra-soft scale $g^{2} T$, i.e. from MQCD}

Ultra-soft physics is not accessible by perturbative methods, due to the unscreened transverse gluonic sector, which would lead to severe infrared problems [1]. This sector is governed by a 
three-dimensional pure gauge theory. Its only parameter is the dimensionful $3 \mathrm{~d}$ gauge coupling $g_{M}^{2}$, which we write as $\hat{g}_{M}^{2} \equiv \frac{N_{\mathrm{c}} g_{M}^{2}}{16 \pi^{2} T}$. The screening length gets generated non-perturbatively, making a numerical lattice Monte-Carlo treatment necessary. The detailed setup for how to incorporate the ultra-soft contribution into the physical pressure by a carefully defined mixture of perturbative and non-perturbative coefficients is explained in detail in Ref. [6]. The result is

$$
\begin{aligned}
\frac{p_{G}(T)}{\mu^{-2 \varepsilon}} & =d_{A} 16 \pi^{2} T^{4} \hat{g}_{M}^{6}\left[8 \alpha_{G}\left(\frac{1}{8 \varepsilon}+L+\ln \frac{N_{\mathrm{c}}^{2}}{4 \pi \hat{g}_{M}^{2}}+1\right)+\frac{1}{3}\right. \\
& +[\text { pert }]-[\mathbf{n s p t}]+[\text { non-pert }]+\mathscr{O}(\varepsilon)],
\end{aligned}
$$

where $d_{A}=N_{\mathrm{c}}{ }^{2}-1$, and $\alpha_{G}=\frac{43}{96}-\frac{157}{6144} \pi^{2}[4,8]$ is a perturbative 4-loop coefficient. The three coefficients enclosed in square brackets originate from measuring the $3 \mathrm{~d}$ YM pressure on the lattice and matching the result to the $\overline{\mathrm{MS}}$ scheme. To be more precise, they are the following.

- The first number stems from a non-perturbative lattice Monte-Carlo measurement of the $3 \mathrm{~d}$ plaquette in pure $\mathrm{SU}\left(N_{\mathrm{c}}\right)$ theory [9],

$$
\begin{aligned}
{[\text { non-pert }] } & =\frac{(4 \pi)^{4}}{8 d_{A} N_{\mathrm{c}}{ }^{6}} \lim _{\beta \rightarrow \infty}\left\{\beta^{4}\left\langle 1-\frac{1}{N_{\mathrm{c}}} \operatorname{Tr} P\right\rangle_{a}-\left[c_{1} \beta^{3}+c_{2} \beta^{2}+c_{3} \beta+c_{4} \ln \beta\right]\right\} \\
& =10.7(4) \text { at } N_{\mathrm{c}}=3,
\end{aligned}
$$

where $\beta=\frac{2 N_{\mathrm{c}}}{g_{M}^{2} a}$ denotes the dimensionless lattice coupling, and $c_{1 . .4}$ are divergences of the $3 \mathrm{~d}$ lattice-regularized plaquette which can be computed in lattice perturbation theory. They read

$$
\begin{aligned}
c_{1} & =\frac{d_{A}}{3}, \\
c_{2} & =\frac{d_{A}}{(4 \pi)^{2}}\left(-\frac{8 \pi^{2}}{9}+5.25449 N_{\mathrm{c}}^{2}\right), \\
c_{3} & =d_{A}\left([0.04978944(1)]+[-0.04289464(7)] N_{\mathrm{c}}{ }^{2}+[0.0147397(3)] N_{\mathrm{c}}{ }^{4}\right) \\
& =6.8612(2) \quad \text { at } N_{\mathrm{c}}=3 \\
c_{4} & =\frac{d_{A} N_{\mathrm{c}}{ }^{6}}{(4 \pi)^{4}} 64 \alpha_{G} .
\end{aligned}
$$

The number in $c_{2}$ is a sum of typical 2-loop (infinite-volume) lattice integrals [10, 9],

$$
-\frac{2}{3}\left(\frac{\Sigma^{2}}{4}-\pi \Sigma-\frac{\pi^{2}}{2}+4 \kappa_{1}+\frac{2}{3} \kappa_{5}\right) \approx 5.25449
$$

with ( $K$ is the complete elliptic integral of first kind) $[11,12,9]$

$$
\begin{aligned}
\Sigma & =\frac{1}{\pi^{2}} \int_{0}^{\pi} \mathrm{d}^{3} x \frac{1}{\sum_{i} \sin ^{2} x_{i}} \\
& =\frac{8}{\pi}(18+12 \sqrt{2}-10 \sqrt{3}-7 \sqrt{6}) K^{2}\left((2-\sqrt{3})^{2}(\sqrt{3}-\sqrt{2})^{2}\right) \approx 3.1759114 \\
\kappa_{1} & =\frac{1}{4 \pi^{4}} \int_{-\pi / 2}^{\pi / 2} \mathrm{~d}^{3} x \mathrm{~d}^{3} y \frac{\sum_{i} \sin ^{2} x_{i} \sin ^{2}\left(x_{i}+y_{i}\right)}{\sum_{i} \sin ^{2} x_{i} \sum_{i} \sin ^{2}\left(x_{i}+y_{i}\right) \sum_{i} \sin ^{2} y_{i}} \approx 0.958382(1) \\
\kappa_{5} & =\frac{1}{\pi^{4}} \int_{-\pi / 2}^{\pi / 2} \mathrm{~d}^{3} x \mathrm{~d}^{3} y \frac{\sum_{i} \sin ^{2} x_{i} \sin ^{2}\left(x_{i}+y_{i}\right) \sin ^{2}\left(y_{i}\right)}{\sum_{i} \sin ^{2} x_{i} \sum_{i} \sin ^{2}\left(x_{i}+y_{i}\right) \sum_{i} \sin ^{2} y_{i}} \approx 1.013041(1)
\end{aligned}
$$


The coefficient $c_{3}$ has been estimated by numerical stochastic perturbation theory (NSPT) for $N_{\mathrm{c}}=3$ [13] and, with higher numerical accuracy and full $N_{\mathrm{c}}$ dependence, computed from 3-loop diagrams in lattice perturbation theory [14]. In principle, it would be nice to know the full $N_{\mathrm{c}^{-}}$ dependence of Eq. (2.2).

- The second number stems from an estimation of (the sum of all) 4-loop vacuum diagrams in lattice perturbation theory by NSPT [15], with the IR divergence regulated by massive gluon- and ghost-propagators (mass term $\frac{m^{2}}{2} A^{2}$ and $m^{2} \bar{c} c$ in the action) [16]. NSPT works on a finite lattice of volume $(a L)^{3}$, so the infinite-volume limit has to be taken first to ensure that the IR is regulated by the mass term only. A very preliminary result is [17]

$$
\begin{aligned}
{[\text { nspt }] } & =\frac{(4 \pi)^{4}}{8 d_{A} N_{\mathrm{c}}{ }^{6}} \lim _{a m \rightarrow 0} \lim _{L \rightarrow \infty}\left\{\left.\left\langle 1-\frac{1}{N_{\mathrm{c}}} \operatorname{Tr} P\right\rangle_{a m}\right|_{\beta^{-4} \text { term }}-c_{4} \ln \frac{1}{a m}\right\} \\
& =\frac{(4 \pi)^{4}}{8 d_{A} N_{\mathrm{c}}{ }^{6}}\left[c_{40}^{\prime}+c_{41}^{\prime} N_{\mathrm{c}}{ }^{2}+c_{42}^{\prime} N_{\mathrm{c}}{ }^{4}+c_{43}^{\prime} N_{\mathrm{c}}{ }^{6}\right] \\
& \approx \frac{4 \pi^{4}}{729} 30 \quad \text { in Feynman gauge at } N_{\mathrm{c}}=3 .
\end{aligned}
$$

To match the precision obtained for [non-pert], this number should be estimated with at least $2 \%$ accuracy. It would be nice to know all four coefficients, in Feynman gauge, either by a direct diagrammatic evaluation, or by doing NSPT for (at least) four different values of $N_{\mathrm{c}}$.

- The third number stems from a matching 4-loop computation in the $(3-2 \varepsilon) \mathrm{d}$ continuum theory, regulated in the IR by gluon- and ghost-masses, with gauge parameter $\xi$. Gauge dependence, introduced by the IR regulator, is guaranteed to cancel against that in $[\mathbf{n s p t}]$. The result reads

$$
\sum[\text { lloop YM vac diags }]=g^{6} d_{A} N_{\mathrm{c}}{ }^{3}\left(\frac{1}{m} J\right)^{4}\left\{\frac{\alpha_{G}}{\varepsilon}+[\text { pert }]+\mathscr{O}(\varepsilon)\right\}
$$

where $J$ is the 1-loop massive tadpole integral $\int_{p} 1 /\left(p^{2}+m^{2}\right)$. We choose Feynman gauge $\xi=1$ which here leads to modified propagators $1 / p^{2} \rightarrow 1 /\left(p^{2}+m^{2}\right)$, and obtain [18]

$$
[\text { pert }]=-3.73134481146281478501 \quad \text { in Feynman gauge }
$$

where the number can be expressed in terms of 18 fully massive 4-loop scalar master integrals $[19,20]$. For general $\xi$, we would have to calculate vacuum diagrams with two mass scales $\left(m^{2}\right.$ and $\xi m^{2}$ ), which presently is beyond our computational capabilities.

The matching condition for the $3 \mathrm{~d}$ gauge coupling reads [21, 22, 23]

$$
\hat{g}_{M}^{2} \equiv \frac{N_{\mathrm{c}} g_{M}^{2}}{16 \pi^{2} T}=\hat{g}_{E}^{2}\left[1-\frac{1}{12} \frac{\hat{g}_{E}^{2}}{\hat{m}_{E}}-\frac{17}{288} \frac{\hat{g}_{E}^{4}}{\hat{m}_{E}^{2}}-\frac{2-\hat{n}}{24} \frac{\hat{g}_{E}^{2} \hat{\lambda}_{E}^{(1)}}{\hat{m}_{E}^{2}}-\frac{3 \hat{n}-1}{24} \frac{\hat{g}_{E}^{2} \hat{\lambda}_{E}^{(2)}}{\hat{m}_{E}^{2}}\right]+\mathscr{O}\left(\frac{\hat{g}_{E}^{8}}{\hat{m}_{E}^{3}}\right),
$$

where $\hat{n} \equiv \frac{N_{\mathrm{c}}{ }^{2}-1}{N_{\mathrm{c}}{ }^{2}}$. For the $g^{6}$ pressure, only the leading coefficient is relevant.

\subsection{Contributions from the soft scale $g T$, i.e. from EQCD}

Soft-scale physics is governed by a three-dimensional gauge theory, coupled to an adjoint Higgs field. This adjoint Higgs theory possesses a small number of dimensionful coupling constants, which are related to the parameters of full QCD (being $g^{2}$ and $T$ ) by the equations given 
below. The contribution of this sector to the pressure is given by

$$
\begin{aligned}
\frac{p_{M}(T)}{\mu^{-2 \varepsilon}} & =d_{A} 16 \pi^{2} T^{4}\left\{\hat{m}_{E}^{3}\left[\frac{1}{3}+\mathscr{O}(\varepsilon)\right]\right. \\
& +\hat{g}_{E}^{2} \hat{m}_{E}^{2}\left[-\frac{1}{4 \varepsilon}+\left(-L+\frac{1}{2} \ln \hat{m}_{E}^{2}+\ln 2-\frac{3}{4}\right)+\mathscr{O}(\varepsilon)\right] \\
& +\hat{g}_{E}^{4} \hat{m}_{E}\left[\left(-\frac{89}{24}-\frac{\pi^{2}}{6}+\frac{11}{6} \ln 2\right)+\mathscr{O}(\varepsilon)\right] \\
& +\hat{g}_{E}^{6}\left[\alpha_{M}\left(\frac{1}{\varepsilon}+8 L-4 \ln \hat{m}_{E}^{2}-8 \ln 2\right)+\beta_{M}+\mathscr{O}(\varepsilon)\right] \\
& +\hat{\lambda}_{E}^{(1)} \hat{m}_{E}^{2}\left[\frac{\hat{n}-2}{4}+\mathscr{O}(\varepsilon)\right]+\hat{\lambda}_{E}^{(2)} \hat{m}_{E}^{2}\left[\frac{1-3 \hat{n}}{4}+\mathscr{O}(\varepsilon)\right] \\
& \left.+\mathscr{O}\left(\hat{g}_{E}^{8} \hat{m}_{E}^{-1}, \hat{\lambda}_{E}^{2} \hat{m}_{E}\right)\right\},
\end{aligned}
$$

with the 4-loop coefficients $\alpha_{M}=\frac{43}{32}-\frac{491}{6144} \pi^{2}, \beta_{M}=-\frac{311}{256}-\frac{43}{32} \ln 2-\frac{19}{6} \ln ^{2} 2+\frac{77}{9216} \pi^{2}-\frac{491}{1536} \pi^{2} \ln 2+$ $\frac{1793}{512} \zeta(3)+\gamma_{10}=-1.391512$ [24], where $\gamma_{10}$ is the leading coefficient of a finite $3 \mathrm{~d}$ scalar 4-loop integral that is known numerically only [24],

$$
\begin{aligned}
\gamma_{10} & =(4 \pi)^{4} \int_{-\infty}^{\infty} \frac{\mathrm{d}^{3} x_{1}}{(2 \pi)^{3}} \frac{\mathrm{d}^{3} x_{2}}{(2 \pi)^{3}} \frac{\mathrm{d}^{3} x_{3}}{(2 \pi)^{3}} \frac{\mathrm{d}^{3} x_{4}}{(2 \pi)^{3}} \frac{1}{\left(x_{1}-x_{3}\right)^{2}} \frac{1}{\left(x_{2}-x_{3}\right)^{2}} \times \\
& \times \frac{1}{x_{1}^{2}+1} \frac{1}{x_{2}^{2}+1} \frac{1}{\left(x_{1}-x_{4}\right)^{2}+1} \frac{1}{\left(x_{2}-x_{4}\right)^{2}+1} \frac{1}{\left(x_{3}-x_{4}\right)^{2}+1} \\
& =0.171007009753(1),
\end{aligned}
$$

and the matching parameters are $[11,3]$

$$
\begin{aligned}
\hat{m}_{E}^{2} \equiv\left(\frac{m_{E}}{4 \pi T}\right)^{2} & =\hat{g}^{2}\left[\tilde{\alpha}_{\mathrm{E} 4}+\left(2 \tilde{\alpha}_{\mathrm{E} 4} L+\tilde{\alpha}_{\mathrm{E} 5}\right) \varepsilon+\mathscr{O}\left(\varepsilon^{2}\right)\right] \\
& +\hat{g}^{4}\left[\left(2 \hat{\beta}_{0} \tilde{\alpha}_{\mathrm{E} 4} L+\tilde{\alpha}_{\mathrm{E} 6}\right)+\left(6 \hat{\beta}_{0} \tilde{\alpha}_{\mathrm{E} 4} L^{2}+\tilde{\beta}_{\mathrm{E} 2}^{(L)} L+\tilde{\beta}_{\mathrm{E} 2}\right) \varepsilon+\mathscr{O}\left(\varepsilon^{2}\right)\right]+\mathscr{O}\left(\hat{g}^{6}\right), \\
\hat{g}_{E}^{2} \equiv \frac{N_{\mathrm{c}} g_{E}^{2}}{16 \pi^{2} T} & =\hat{g}^{2}+\hat{g}^{4}\left[\left(2 \hat{\beta}_{0} L+\tilde{\alpha}_{\mathrm{E} 7}\right)+\left(2 \hat{\beta}_{0} L^{2}+2 \tilde{\alpha}_{\mathrm{E} 7} L+\tilde{\beta}_{\mathrm{E} 3}\right) \varepsilon+\mathscr{O}\left(\varepsilon^{2}\right)\right] \\
& +\hat{g}^{6}\left[4 \hat{\beta}_{0}^{2} L^{2}+2\left(\hat{\beta}_{1}+2 \hat{\beta}_{0} \tilde{\alpha}_{\mathrm{E} 7}\right) L+\tilde{\gamma}_{\mathrm{E} 1}+\mathscr{O}(\varepsilon)\right]+\mathscr{O}\left(\hat{g}^{8}\right), \\
\hat{\lambda}_{E}^{(1)} \equiv \frac{N_{\mathrm{c}}{ }^{2} \lambda_{E}^{(1)}}{16 \pi^{2} T} & =\hat{g}^{4}[4+\mathscr{O}(\varepsilon)]+\mathscr{O}\left(\hat{g}^{6}\right) \\
\hat{\lambda}_{E}^{(2)} \equiv \frac{N_{\mathrm{c}} \lambda_{E}^{(2)}}{16 \pi^{2} T} & =\hat{g}^{4}\left[\frac{4}{3}(1-z)+\mathscr{O}(\varepsilon)\right]+\mathscr{O}\left(\hat{g}^{6}\right),
\end{aligned}
$$

where we have used the beta-function coefficients $\hat{\beta}_{0}=\frac{11-2 z}{3}, \hat{\beta}_{1}=\frac{34}{3}-\frac{10}{3} z-z \hat{n}$ and, for brevity, set $z \equiv N_{\mathrm{f}} / N_{\mathrm{c}}$. The coefficients read [3, 25, 26]

$$
\begin{aligned}
& \tilde{\alpha}_{\mathrm{E} 4}=\frac{2+z}{6}, \\
& \tilde{\alpha}_{\mathrm{E} 5}=2 \tilde{\alpha}_{\mathrm{E} 4} Z_{1}+\frac{z}{6}(1-2 \ln 2), \\
& \tilde{\alpha}_{\mathrm{E} 6}=\frac{1}{3} \tilde{\alpha}_{\mathrm{E} 4}\left(6 \hat{\beta}_{0} \gamma_{0}+5+2 z-8 z \ln 2\right)-\frac{z}{2} \hat{n},
\end{aligned}
$$




$$
\tilde{\alpha}_{\mathrm{E} 7}=2 \hat{\beta}_{0} \gamma_{0}+\frac{1}{3}-\frac{8}{3} z \ln 2
$$

as well as $[27,23]$

$$
\begin{aligned}
\tilde{\beta}_{\mathrm{E} 2}^{(L)} & =4 \hat{\beta}_{0} \tilde{\alpha}_{\mathrm{E} 4}\left(2 \gamma_{0}+Z_{1}\right)+\frac{1}{9}\left(20+29 z+2 z^{2}\right) \\
& -2 z(\hat{n}+3 \ln 2)-\frac{4}{3} z^{2} \ln 2 \\
\tilde{\beta}_{\mathrm{E} 2} & =\frac{1}{4} \hat{\beta}_{0} \tilde{\alpha}_{\mathrm{E} 4}\left(+\pi^{2}-16 \gamma_{1}\right)+\frac{2}{3} \tilde{\alpha}_{\mathrm{E} 4} Z_{1}\left(6 \hat{\beta}_{0} \gamma_{0}+5+2 z-8 z \ln 2\right) \\
& +\frac{2}{9} \gamma_{0}(5+10 z-(19+2 z) z \ln 2)+\frac{2}{9}+\frac{z}{18}\left(7+6 \ln 2-16 \ln ^{2} 2\right) \\
& +\frac{z^{2}}{9}\left(1-2 \ln 2+4 \ln ^{2} 2\right)-\frac{z}{6} \hat{n}\left(3+6 \gamma_{0}+6 Z_{1}+10 \ln 2\right), \\
\tilde{\beta}_{\mathrm{E} 3} & =\left(\frac{\pi^{2}}{4}-4 \gamma_{1}\right) \hat{\beta}_{0}+\frac{2}{3} \gamma_{0}-\frac{8}{3} \ln 2\left(\ln 2+2 \gamma_{0}\right) z, \\
\tilde{\gamma}_{\mathrm{E} 1} & =2 \hat{\beta}_{1} \gamma_{0}+\tilde{\alpha}_{\mathrm{E} 7}^{2}+\frac{341}{18}-\frac{10}{9} \zeta(3) \\
& -\frac{z}{9}(43+24 \ln 2+5 \zeta(3))-\frac{z}{12} \hat{n}(23+80 \ln 2-14 \zeta(3)),
\end{aligned}
$$

where the $\gamma_{n}$ are expansion coefficients of the Zeta function $\zeta(1-\varepsilon)=-\frac{1}{\varepsilon}+\sum_{n=0}^{\infty} \frac{\varepsilon^{n}}{n !} \gamma_{n}$ (note that $\left.\gamma_{0} \equiv \gamma_{E}=0.577216\right)$. For the $g^{6}$ pressure, the $\hat{g}^{6}$ terms of Eq. (2.20) are irrelevant.

\subsection{Contributions from the hard scale $2 \pi T$, i.e. from thermal QCD}

Hard-scale physics can be treated perturbatively in naive thermal QCD, meaning a simple $g^{2}$ expansion, without the need for resummations, thermal masses, or hard thermal loops. This is due to all IR effects being properly incorporated into EQCD and MQCD, and is one of the main conceptual advantages of using the effective theory setup. The contribution to the pressure from hard momentum scales reads

$$
\begin{aligned}
\frac{p_{E}(T)}{\mu^{-2 \varepsilon}} & =d_{A} 16 \pi^{2} T^{4} \frac{1}{16} \frac{1}{45}\left\{\tilde{\alpha}_{\mathrm{E} 1}+\hat{g}^{2}\left[\tilde{\alpha}_{\mathrm{E} 2}+\mathscr{O}(\varepsilon)\right]\right. \\
& +\hat{g}^{4}\left[\tilde{\alpha}_{\mathrm{E} 4} \frac{180}{\varepsilon}+\left(180 \cdot 6 \tilde{\alpha}_{\mathrm{E} 4}+2 \hat{\beta}_{0} \tilde{\alpha}_{\mathrm{E} 2}\right) L+\tilde{\alpha}_{\mathrm{E} 3}+\mathscr{O}(\varepsilon)\right] \\
& \left.+\hat{g}^{6}\left[\frac{\tilde{\beta}_{\mathrm{E} 1}^{(\mathrm{div})}}{\varepsilon}+\tilde{\beta}_{\mathrm{E} 1}^{\left(L^{2}\right)} L^{2}+\tilde{\beta}_{\mathrm{E} 1}^{(L)} L+\tilde{\beta}_{\mathrm{E} 1}+\mathscr{O}(\varepsilon)\right]+\mathscr{O}\left(\hat{g}^{8}\right)\right\},
\end{aligned}
$$

with ideal-gas coefficient $\tilde{\alpha}_{\mathrm{E} 1}=1+\frac{7}{4} \overline{\hat{n}}, \tilde{\alpha}_{\mathrm{E} 2}=-\frac{5}{4}(4+5 z)[28,29]$ and, writing $Z_{1} \equiv \frac{\zeta^{\prime}(-1)}{\zeta(-1)}$ and $Z_{3} \equiv \frac{\zeta^{\prime}(-3)}{\zeta(-3)},[30]$

$$
\begin{aligned}
\tilde{\alpha}_{\mathrm{E} 3} & =180\left(\tilde{\alpha}_{\mathrm{E} 4}\right)^{2} \gamma_{0}+5\left[\left(\frac{116}{5}+\frac{220}{3} Z_{1}-\frac{38}{3} Z_{3}\right)\right. \\
& +\frac{z}{2}\left(\frac{1121}{60}-\frac{157}{5} \ln 2+\frac{146}{3} Z_{1}-\frac{1}{3} Z_{3}\right) \\
& \left.+\frac{z^{2}}{4}\left(\frac{1}{3}-\frac{88}{5} \ln 2+\frac{16}{3} Z_{1}-\frac{8}{3} Z_{3}\right)+\frac{z}{4} \hat{n}\left(\frac{105}{4}-24 \ln 2\right)\right],
\end{aligned}
$$


and unknown coefficients $\beta_{\mathrm{E} 1}$, which can be determined e.g. by a 4-loop computation of vacuum diagrams in thermal QCD. Since $p_{\mathrm{QCD}}$ is physical, the divergent and scale-dependent parts of $\beta_{\mathrm{E} 1}$ are related to the other coefficients introduced in the above, serving as a valuable check on this open computation. Specifically, from 2-loop running of the $4 \mathrm{~d}$ gauge coupling

$$
\hat{g}^{2} \equiv \frac{N_{\mathrm{c}} g^{2}(\bar{\mu})}{16 \pi^{2}}=\hat{g}^{2}\left(\bar{\mu}_{0}\right)+\hat{g}^{4}\left(\bar{\mu}_{0}\right)\left(-2 \hat{\beta}_{0} \ell\right)+\hat{g}^{6}\left(\bar{\mu}_{0}\right)\left(4 \hat{\beta}_{0}^{2} \ell^{2}-2 \hat{\beta}_{1} \ell\right),
$$

where $\ell \equiv \ln \frac{\bar{\mu}}{\bar{\mu}_{0}}=L-\ln \frac{\bar{\mu}_{0}}{4 \pi T}$, one can already fix

$$
\begin{aligned}
\tilde{\beta}_{\mathrm{E} 1}^{(\mathrm{div})} & =180\left[4 \hat{\beta}_{0} \tilde{\alpha}_{\mathrm{E} 4} L+\tilde{\alpha}_{\mathrm{E} 6}+\tilde{\alpha}_{\mathrm{E} 4} \tilde{\alpha}_{\mathrm{E} 7}-4\left(\alpha_{G}+\alpha_{M}\right)\right], \\
\tilde{\beta}_{\mathrm{E} 1}^{\left(L^{2}\right)} & =180\left[28 \hat{\beta}_{0} \tilde{\alpha}_{\mathrm{E} 4}\right]+4 \hat{\beta}_{0}^{2} \tilde{\alpha}_{\mathrm{E} 2}, \\
\tilde{\beta}_{\mathrm{E} 1}^{(L)} & =180\left[4 \tilde{\alpha}_{\mathrm{E} 6}+8 \tilde{\alpha}_{\mathrm{E} 4} \tilde{\alpha}_{\mathrm{E} 7}-2 \hat{\beta}_{0} \tilde{\alpha}_{\mathrm{E} 5}-32\left(\alpha_{G}+\alpha_{M}\right)+\tilde{\beta}_{\mathrm{E} 2}^{(L)}\right] \\
& +2 \hat{\beta}_{1} \tilde{\alpha}_{\mathrm{E} 2}+4 \hat{\beta}_{0} \tilde{\alpha}_{\mathrm{E} 3} .
\end{aligned}
$$

The remaining $g^{6}$-coefficient, $\tilde{\beta}_{\mathrm{E} 1}$ however, entails a four-loop computation of all connected vacuum diagrams involving quarks, gluons and ghosts, a computation that has so far not been tackled due to the formidable task of solving many genuine 4-loop sum-integrals. From diagrammatic arguments, it is clearly a polynomial in $z=N_{\mathrm{f}} / N_{\mathrm{c}}$,

$$
\tilde{\beta}_{\mathrm{E} 1}=\#_{0}+z \#_{1}+z^{2} \#_{2}+z^{3} \#_{3},
$$

and we will in the following indicate how two of its coefficients (the first and last) can be crudely estimated numerically already.

\section{Putting everything together}

Expanding in $\varepsilon$, all poles cancel, as they should. In practice we make use of Eqs. (2.15),(2.19) and (2.20) to re-expand all terms with a factor $1 / \varepsilon$ or $L$ in Eqs. (2.1) and (2.16) in terms of $\hat{g}^{2}$. After cancellation of the poles (and taking into account terms like $\frac{1}{\varepsilon} \cdot \varepsilon$ ), we can now take the limit $\varepsilon \rightarrow 0$ in Eqs. (2.19), (2.20), whence

$$
\begin{aligned}
\hat{m}_{E}^{2} & =\hat{g}^{2} \tilde{\alpha}_{\mathrm{E} 4}+\hat{g}^{4}\left[2 \hat{\beta}_{0} \tilde{\alpha}_{\mathrm{E} 4} L+\tilde{\alpha}_{\mathrm{E} 6}\right]+\mathscr{O}\left(\hat{g}^{6}\right), \\
\hat{g}_{E}^{2} & =\hat{g}^{2}+\hat{g}^{4}\left[2 \hat{\beta}_{0} L+\tilde{\alpha}_{\mathrm{E} 7}\right]+\hat{g}^{6}\left[4 \hat{\beta}_{0}^{2} L^{2}+2\left(\hat{\beta}_{1}+2 \hat{\beta}_{0} \tilde{\alpha}_{\mathrm{E} 7}\right) L+\tilde{\gamma}_{\mathrm{E} 1}\right]+\mathscr{O}\left(\hat{g}^{8}\right) .
\end{aligned}
$$

Collecting explicit logarithms $L$, they precisely cancel the scale dependence of $\hat{g}^{2}$ up to the order of the computation, and can hence be absorbed by writing

$$
\tilde{g}^{2}=\hat{g}^{2}+\hat{g}^{4} 2 \hat{\beta}_{0} L+\hat{g}^{6}\left(4 \hat{\beta}_{0}^{2} L^{2}+2 \hat{\beta}_{1} L\right)+\mathscr{O}\left(\hat{g}^{8}\right) .
$$

Note that this coupling is explicitly scale independent to the order we are working, $\partial_{\ln \bar{\mu}^{2}} \tilde{g}^{2}=\mathscr{O}\left(\tilde{g}^{8}\right)$. We now have the full pressure as a sum of its ultra-soft, soft and hard parts as

$$
p_{\mathrm{QCD}}=d_{A} \pi^{2} T^{4}\left\{16 \hat{p}_{\mathrm{us}}+16 \hat{p}_{\mathrm{s}}+\frac{1}{45} \hat{p}_{\mathrm{h}}\right\},
$$



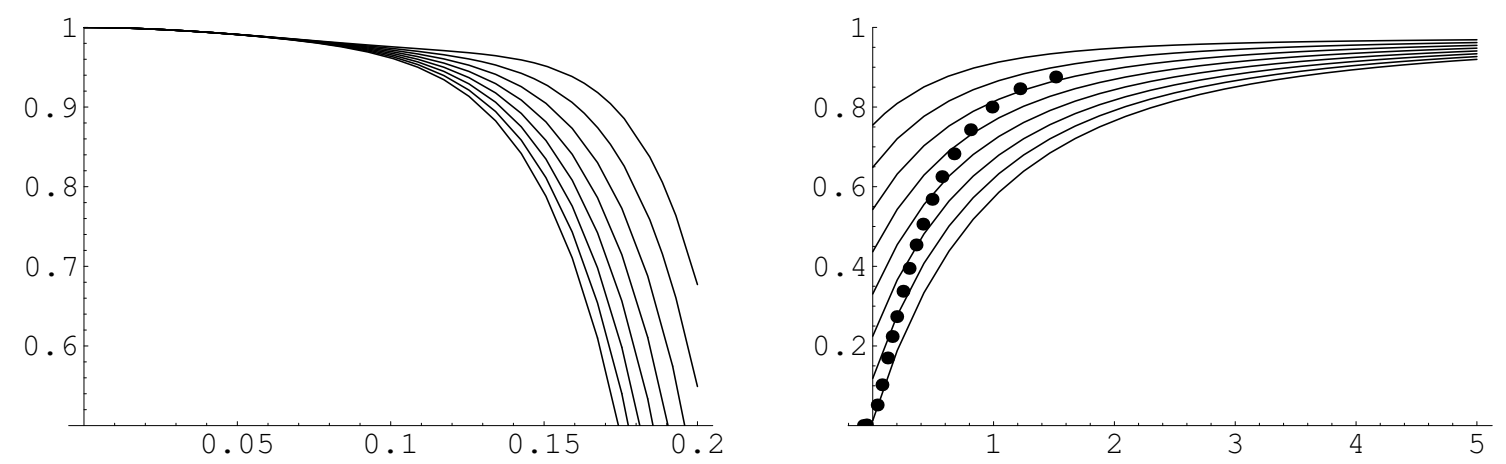

Figure 1: Left panel: The normalized QCD pressure $p_{\mathrm{QCD}} / p_{\mathrm{SB}}$ at $N_{\mathrm{f}}=0$ plotted versus the effective coupling $\tilde{g}$ from Eq. (3.3). The $\tilde{g}^{6}$ coefficient depends on an unknown parameter $\Delta$ as defined in Eq. (3.8), and the different curves correspond to choosing $\Delta=-2000$ (lowest curve) to $\Delta=+12000$, in steps of 2000. Left panel: The same, plotted versus $\ln \frac{T}{T_{C}}$. The black dots correspond to lattice data from [31].

$$
\begin{aligned}
\hat{p}_{\mathrm{us}} & =\hat{g}_{M}^{6}\left[8 \alpha_{G}\left(\ln \frac{N_{\mathrm{c}}^{2}}{4 \pi \hat{g}_{M}^{2}}+1\right)+\frac{1}{3}+[\text { pert }]-[\mathbf{n s p t}]+[\text { non-pert }]\right] \\
\hat{p}_{\mathrm{s}} & =\hat{m}_{E}^{3} \frac{1}{3}+\hat{g}_{E}^{2} \hat{m}_{E}^{2}\left[\ln \left(2 \hat{m}_{E}\right)-\frac{3}{4}\right]+\hat{g}_{E}^{4} \hat{m}_{E}\left[-\frac{89}{24}-\frac{\pi^{2}}{6}+\frac{11}{6} \ln 2\right] \\
& +\hat{g}_{E}^{6}\left[\beta_{M}-8 \alpha_{M} \ln \left(2 \hat{m}_{E}\right)\right]+\hat{\lambda}_{E}^{(1)} \hat{m}_{E}^{2} \frac{\hat{n}-2}{4}+\hat{\lambda}_{E}^{(2)} \hat{m}_{E}^{2} \frac{1-3 \hat{n}}{4} \\
\hat{p}_{\mathrm{h}} & =\tilde{\alpha}_{\mathrm{E} 1}+\tilde{g}^{2} \tilde{\alpha}_{\mathrm{E} 2}+\tilde{g}^{4}\left[\tilde{\alpha}_{\mathrm{E} 3}-180 \tilde{\alpha}_{\mathrm{E} 5}\right]+\tilde{g}^{6}\left[\tilde{\beta}_{\mathrm{E} 1}-180\left(\tilde{\beta}_{\mathrm{E} 2}+\tilde{\alpha}_{\mathrm{E} 4} \tilde{\beta}_{\mathrm{E} 3}+\tilde{\alpha}_{\mathrm{E} 5} \tilde{\alpha}_{\mathrm{E} 7}\right)\right]
\end{aligned}
$$

The $g^{6}$ coefficient of $p_{\mathrm{QCD}}$ hence depends on a constant

$$
\Delta \equiv \tilde{\beta}_{\mathrm{E} 1} \pm 720 \delta_{\mathrm{NP}} \pm 384.826 \delta_{\mathrm{NSPT}}
$$

where we recall that $\tilde{\beta}_{\mathrm{E} 1}$ stands for the result of the open 4-loop computation, and the $\delta$ parameterize the error-bars of the numerical constants from Eqs. $(2.3,2.13)$ as $\left[10.7 \pm \delta_{\mathrm{NP}}\right]$ and $\left[30 \pm \delta_{\mathrm{NSPT}}\right]$, respectively. Assuming that the NSPT computation will finally have an error-bar of about $2 \%$, which is comparable in precision to the lattice error-bar $\delta_{\mathrm{NP}}=0.4, \Delta=\tilde{\beta}_{\mathrm{E} 1} \pm 600$. In the following, we will for simplicity set $\delta_{\mathrm{NP}}=\delta_{\mathrm{NSPT}}=0$, remembering the induced error-bar on $\tilde{\beta}_{\mathrm{E} 1}$. Using the same coupling as in $p_{\mathrm{h}}$, the above matching conditions now read

$$
\begin{aligned}
\hat{m}_{E}^{2} & =\tilde{g}^{2} \tilde{\alpha}_{\mathrm{E} 4}+\tilde{g}^{4} \tilde{\alpha}_{\mathrm{E} 6}+\mathscr{O}\left(\tilde{g}^{6}\right), \\
\hat{g}_{E}^{2} & =\tilde{g}^{2}+\tilde{g}^{4} \tilde{\alpha}_{\mathrm{E} 7}+\tilde{g}^{6} \tilde{\gamma}_{\mathrm{E} 1}+\mathscr{O}\left(\tilde{g}^{8}\right), \\
\hat{\lambda}_{E}^{(1)} & =\tilde{g}^{4} 4+\mathscr{O}\left(\tilde{g}^{6}\right), \\
\hat{\lambda}_{E}^{(2)} & =\tilde{g}^{4} \frac{4}{3}(1-z)+\mathscr{O}\left(\tilde{g}^{6}\right) .
\end{aligned}
$$

We would now like to plot the result for $p_{\mathrm{QCD}}$. Identifying the non-interacting (ideal-gas;

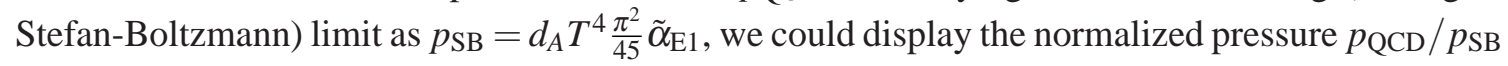
as a function of the coupling $\tilde{g}$, for fixed $N_{\mathrm{f}}$. This is done in the left panel of Fig. 1 , at $N_{\mathrm{f}}=0$ and for various $\Delta$. Our goal, however, should be to try to make contact to existing lattice determinations of the full pressure, where typically $p_{\mathrm{QCD}} / p_{\mathrm{SB}}$ is given as a function of $T / T_{c}$. Note that continuumextrapolated lattice data exist for $N_{\mathrm{f}}=0$ only, so in the following we will restrict to this special case. 

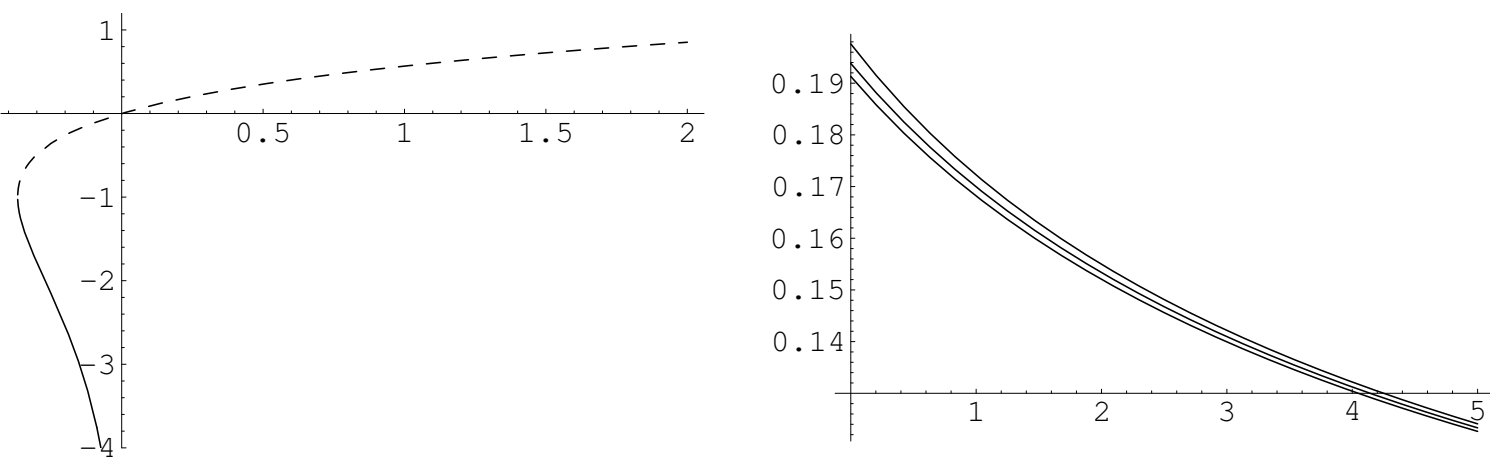

Figure 2: Left panel: The two real branches of $W(z)$ vs $z$. The upper (dashed) branch is $W_{0}(z)$, the lower (solid) branch is $W_{-1}(z)$. Right panel: The effective coupling $\tilde{g}$ from Eq. (3.3) plotted versus $\ln \frac{T}{T_{c}}$, using the choices explained in Sec. 3. The upper/lower curve corresponds to the uncertainty in scale choice stemming from fixing $\bar{\mu}$ and determining $T_{c} / \Lambda_{\overline{\mathrm{MS}}}$ with $\left(\delta_{\mu}, \delta_{T_{c}}\right)=(2.0,0.9) /(0.8,1.1)$, the bigger effect coming from the latter parameter.

Aiming for this rather phenomenological comparison, we evidently need to make some choices, specified below.

We use the running $4 \mathrm{~d}$ coupling from the exact solution of the 2-loop RGE equation,

$$
\hat{g}^{2}(\bar{\mu})=\frac{-\hat{\beta}_{0} / \hat{\beta}_{1}}{1+W_{-1}\left(-\frac{\hat{\beta}_{0}^{2}}{\hat{\beta}_{1}} \exp \left[-1-2 \frac{\hat{\beta}_{0}^{2}}{\hat{\beta}_{1}}\left(L+\ln \frac{4 \pi T}{\Lambda_{\overline{\mathrm{MS}}}}\right)\right]\right)} .
$$

Here, $W_{-1}(z)$ is one of the two real branches of the Lambert W function (see e.g. [32] and the left panel of Fig. 2; $W(z)$ is the function that satisfies $W \exp (W)=z$ ). Note that the above solution entails two choices: The branch of the $W$-function and the constant were chosen in accord with asymptotic freedom (note that the argument of $\mathrm{W} \rightarrow 0^{-}$for $\bar{\mu} \rightarrow \infty$ ) and the 'usual' definition of $\Lambda_{\overline{\mathrm{MS}}}$ (being the absence of a $1 / \ln ^{2} \bar{\mu}$ term in the asymptotic expansion of $\hat{g}(\bar{\mu})$ at large $\bar{\mu}$ ).

Although in principle all dependence on the renormalization scale $\bar{\mu}$, entering through $L$, is of higher order, in practice we need to fix it once we need numerical values for the coupling $\tilde{g}$. Following [23], we choose the scale $\bar{\mu}$ by the principle of minimal sensitivity applied to the 1-loop result for $\hat{g}_{E}^{2}$, and then estimate the scale-dependence by a variation of a factor of $\delta_{\mu}=$ [0.8..2.0] around this $\bar{\mu}_{\text {opt }}$, obtaining $L=-\frac{\tilde{\alpha}_{\mathrm{E} 7}}{2 \hat{\beta}_{0}}+\ln \frac{\bar{\mu}}{\bar{\mu}_{\text {opt }}}$. The slightly asymmetric choice of $\delta_{\mu}$ here reflects the fact that the 1-loop $\hat{g}_{E}^{2}$ falls off more steeply on one side of the plateau than the other.

To compare with continuum-extrapolated lattice data [31], we use $\frac{T_{c}}{\Lambda_{\mathrm{MS}}}=1.22 \delta_{T_{c}}$ where $\delta_{T_{c}}=$ [0.9..1.1] encompasses the central values and error bars of estimates of this quantity from different lattice collaborations (for a summary of the different methods and results, see [23]). This would translate into a horizontal error bar for the lattice data when plotted against $T / \Lambda_{\overline{\mathrm{MS}}}$.

In the right panel of Fig. 2, we have plotted the effective coupling $\tilde{g}$ as defined in Eq. (3.3), converted to a function of $\ln \frac{T}{T_{c}}$. Note that its value is smaller than 0.2 even at $T_{c}$.

The normalized pressure $p_{\mathrm{QCD}} / p_{\mathrm{SB}}$, converted to a function of $\ln \frac{T}{T_{c}}$ along the lines above, is displayed in the right panel of Fig. 1. For comparison, the continuum-extrapolated lattice data of Ref. [31] has been included as black dots. The figure suggests a value for the $N_{\mathrm{f}}=0$ coefficient of the unknown constant $\tilde{\beta}_{\mathrm{E} 1}, \#_{0} \approx 8500 \pm 600$, bearing in mind the error bar defined in Eq. (3.8). 


\section{Discussion}

We have currently no idea what the 4-loop hard-scale coefficient $\tilde{\beta}_{\mathrm{E} 1}$ is, even though it can be computed diagrammatically. As already mentioned above, it should be a polynomial in $z=N_{\mathrm{f}} / N_{\mathrm{c}}$, $\tilde{\beta}_{\mathrm{E} 1}=\#_{0}+z \#_{1}+z^{2} \#_{2}+z^{3} \#_{3}$, where only a single (ring) diagram contributes to $\#_{3}$, suggesting it as the first test-case for 4-loop sum-integral technology.

It seems possible to give an estimate of the highest- $N_{\mathrm{f}}$ contribution to $\tilde{\beta}_{\mathrm{E} 1}$ from the large- $N_{\mathrm{f}}$ solution for the pressure, since terms of order $g^{6} N_{\mathrm{f}}^{3}$ originate from the hard-scale pressure $p_{\mathrm{E}}$ only. Indeed, in [33] this was attempted by fitting the numerically known exact large- $N_{\mathrm{f}}$ pressure with a polynomial in $g$. This results in $\#_{3}=\frac{45}{8 \pi^{2}}[+20(2)]-\frac{20}{9} \ln 2\left(1+12 \gamma_{0}-\frac{364}{5} \ln 2+16 Z_{1}-8 Z_{3}\right) \approx$ $[+36(1)]$, where the terms proportional to $\ln 2$ originate from translating the choice of renormalization scale $\bar{\mu}=\pi T$ of [33] to our definition of $\tilde{\beta}_{\mathrm{E} 1}$, where powers of $L=\ln \frac{\bar{\mu}}{4 \pi T}$ were subtracted out.

Furthermore, fitting the full $g^{6}$ pressure at $N_{\mathrm{f}}=0$ and $N_{\mathrm{c}}=3$ to lattice data around $4 T_{c}$ [31] suggests a value $\#_{0} \approx 8500 \pm 600$, if one takes the conjecture for granted that all higher-order corrections sum up to a subdominant contribution. There is no guarantee whatsoever that this conjecture holds, making a perturbative computation of the $\#_{i}$ unavoidable. We take the above check against the lattice data as indication that the effective theory setup has a chance to analytically describe the transition from temperatures as low as a few times $T_{c}$ to infinite temperatures, in terms of computable corrections to the ideal-gas limit.

\section{Acknowledgments}

Y.S. would like to thank the organizers for a very enjoyable workshop, and especially A. Patkós for sharing his insights into Hungarian art.

\section{References}

[1] A. D. Linde, Phys. Lett. B 96 (1980) 289;

D. J. Gross, R. D. Pisarski and L. G. Yaffe, Rev. Mod. Phys. 53 (1981) 43.

[2] T. Appelquist and R. D. Pisarski, Phys. Rev. D 23 (1981) 2305.

[3] E. Braaten and A. Nieto, Phys. Rev. Lett. 76 (1996) 1417 [hep-ph/9508406];

E. Braaten and A. Nieto, Phys. Rev. D 53 (1996) 3421 [hep-ph/9510408].

[4] K. Kajantie, M. Laine, K. Rummukainen and Y. Schröder, Phys. Rev. D 67 (2003) 105008 [hep-ph/0211321].

[5] J. I. Kapusta, Nucl. Phys. B 148, 461 (1979).

[6] M. Laine and Y. Schröder, hep-ph/0603048.

[7] C. P. Korthals Altes, R. D. Pisarski and A. Sinkovics, Phys. Rev. D 61 (2000) 056007 [hep-ph/9904305];

J. P. Blaizot, E. Iancu and A. Rebhan, Phys. Lett. B 523, 143 (2001) [hep-ph/0110369];

A. Vuorinen, Phys. Rev. D 67 (2003) 074032 [hep-ph/0212283];

A. Vuorinen, Phys. Rev. D 68 (2003) 054017 [hep-ph/0305183];

A. Hart, M. Laine and O. Philipsen, Nucl. Phys. B 586 (2000) 443 [hep-ph/0004060]. 
[8] Y. Schröder, Nucl. Phys. Proc. Suppl. 129 (2004) 572 [hep-lat/0309112].

[9] A. Hietanen, K. Kajantie, M. Laine, K. Rummukainen and Y. Schröder, JHEP 0501 (2005) 013 [hep-lat/0412008].

[10] U. M. Heller and F. Karsch, Nucl. Phys. B 251 (1985) 254.

[11] K. Farakos, K. Kajantie, K. Rummukainen and M. E. Shaposhnikov, Nucl. Phys. B 442 (1995) 317 [hep-lat/9412091].

[12] M. Laine and A. Rajantie, Nucl. Phys. B 513 (1998) 471 [hep-lat/9705003].

[13] F. Di Renzo, A. Mantovi, V. Miccio and Y. Schröder, JHEP 0405 (2004) 006 [hep-lat/0404003].

[14] H. Panagopoulos, A. Skouroupathis and A. Tsapalis, hep-lat/0601009.

[15] F. Di Renzo and L. Scorzato, JHEP 0410 (2004) 073 [hep-lat/0410010].

[16] C. Torrero, F. Di Renzo, V. Miccio, M. Laine and Y. Schröder, Proc. Sci. LAT2005 (2005) 189 [hep-lat/0509157].

[17] C. Torrero, PhD thesis 2006, Parma University, Italy (unpublished).

[18] Y. Schröder, unpublished.

[19] Y. Schröder, Nucl. Phys. Proc. Suppl. 116 (2003) 402 [hep-ph/0211288].

[20] Y. Schröder and A. Vuorinen, hep-ph/0311323.

[21] K. Farakos, K. Kajantie, K. Rummukainen and M. E. Shaposhnikov, Nucl. Phys. B 425 (1994) 67 [hep-ph/9404201].

[22] P. Giovannangeli, Phys. Lett. B 585 (2004) 144 [hep-ph/0312307];

P. Giovannangeli, Nucl. Phys. B 738 (2006) 23 [hep-ph/0506318].

[23] M. Laine and Y. Schröder, JHEP 0503 (2005) 067 [hep-ph/0503061].

[24] K. Kajantie, M. Laine, K. Rummukainen and Y. Schröder, JHEP 0304 (2003) 036 [hep-ph/0304048].

[25] K. Kajantie, M. Laine, K. Rummukainen and M. E. Shaposhnikov, Nucl. Phys. B 458 (1996) 90 [hep-ph/9508379].

[26] S. z. Huang and M. Lissia, Nucl. Phys. B 438 (1995) 54 [hep-ph/9411293];

S. z. Huang and M. Lissia, Nucl. Phys. B 480 (1996) 623 [hep-ph/9511383].

[27] Y. Schröder, hep-ph/0410130.

[28] E. V. Shuryak, Sov. Phys. JETP 47 (1978) 212 [Zh. Eksp. Teor. Fiz. 74 (1978) 408].

[29] S. A. Chin, Phys. Lett. B 78 (1978) 552.

[30] P. Arnold and C. X. Zhai, Phys. Rev. D 50 (1994) 7603 [hep-ph/9408276];

P. Arnold and C. x. Zhai, Phys. Rev. D 51 (1995) 1906 [hep-ph/9410360].

[31] G. Boyd, J. Engels, F. Karsch, E. Laermann, C. Legeland, M. Lütgemeier and B. Petersson, Nucl. Phys. B 469 (1996) 419 [hep-lat/9602007].

[32] R. M. Corless, G. H. Gonnet, D. E. G. Hare, D. J. Jeffrey, and D. E. Knuth, "On the Lambert W Function", Advances in Computational Mathematics, volume 5, 1996, pp. 329-359.

[33] A. Ipp and A. Rebhan, JHEP 0306 (2003) 032 [hep-ph/0305030]. 